\title{
Linx
}

Revue des linguistes de l'université Paris X Nanterre

$59 \mid 2008$

Les conjonctions en diachronie : parcours sémantiques

\section{Françoise Boch et Fanny Rinck (sous la direction de), Lidil n 41, Mai 2010 : Enonciation et rhétorique dans l'écrit scientifique}

Irène Fenoglio

\section{(2) OpenEdition}

\section{Journals}

Édition électronique

URL : http://journals.openedition.org/linx/646

DOI : $10.4000 /$ linx.646

ISSN : 2118-9692

Éditeur

Presses universitaires de Paris Nanterre

Édition imprimée

Date de publication : 1 décembre 2008

Pagination : 185-187

ISSN : 0246-8743

Référence électronique

Irène Fenoglio, "Françoise Boch et Fanny Rinck (sous la direction de), Lidil n 41, Mai 2010

Enonciation et rhétorique dans l'écrit scientifique », Linx [En ligne], 59| 2008, mis en ligne le 08 juillet

2011, consulté le 22 septembre 2020. URL : http://journals.openedition.org/linx/646 ; DOI : https:// doi.org/10.4000/linx.646

Ce document a été généré automatiquement le 22 septembre 2020.

Département de Sciences du langage, Université Paris Ouest 


\title{
Françoise Boch et Fanny Rinck (sous la direction de), Lidil $n^{\circ} 41$, Mai 2010 : Enonciation et rhétorique dans l'écrit scientifique
}

\author{
Irène Fenoglio
}

\section{RÉFÉRENCE}

Françoise Boch et Fanny Rinck (sous la direction de), Lidil nº 41, Mai 2010 : Enonciation et rhétorique dans l'écrit scientifique.

1 Les huit articles de ce volume, dense, parmi lesquels nous faisons entrer l'introduction des éditrices qui proposent un état des lieux de la question, portent sur le discours scientifique entendu comme « discours produit dans le cadre de l'activité de recherche à des fins de construction et de diffusion du savoir » (p. 5). L'introduction à l'ensemble insiste sur le développement actuel de ce champ aussi bien dans une approche de linguistique descriptive que dans une logique de constitution de corpus. Deux traits saillants ressortent qui seront abordés à des échelles diverses dans tous les articles: l'article scientifique est le genre privilégié dans ce type d'étude et l'analyse linguistique $\mathrm{du}$ positionnement de l'auteur dans un écrit scientifique demeure une question permanente.

2 L'intérêt majeur de ce numéro de Lidil est de faire se croiser, à travers des articles diversifiés par leurs corpus et leurs programmes, une série de questions récurrentes sous différents angles de vue.

3 Les corpus analysés proposent déjà, par leur constitution même, différentes interrogations. En effet, ils sont diversifiés selon plusieurs perspectives. 
4 - Ils portent sur différentes langues : le français, bien sûr (A. Tutin, K. Fløtum et E. T. Vold, U. Reutner), l'anglais (F. Salager-Meyer, M. A Alcaraz-Ariza et M. Pabon, I. Kanté), l'hébreu (Z. Livnat), faisant émerger des éléments linguistiques spécifiques.

- Ils portent sur différents genres d'écrits scientifiques: articles où sont abordés différentes questions sur lesquelles nous reviendrons et où, par exemple, Z. Livnat identifie les différentes formes de construction impersonnelles à la charnière entre syntaxe et sémantique, recensions d'ouvrages (F. Salager, M. A. Alcaraz Ariza et M. Pabon) et divers autres (C. Donahue).

- Ils portent sur différentes disciplines, même si les articles linguistiques semblent privilégiés : linguistique, psychologie, droit mais aussi contrastivement sciences dures (c. Donahue)

- Enfin les corpus sont pris le plus souvent en synchronie mais aussi en diachronie: F. Salager, M. A. Alcaraz Ariza et M. Pabon mettent en valeur, dans la recension d'ouvrages, genre très différent de celui de l'article, le fait que les formes d'identifications d'auteur y sont devenues, depuis le $\mathrm{XIX}^{\mathrm{e}}$ siècle, de plus en plus succinctes, alors que l'auteur de la recension lui-même s'identifie de plus en plus subjectivement en $I$.

5 Les méthodologies sont également diversifiées: analyses internes et analyses contrastives. Les analyses contrastives sont menées entre diverses disciplines, entre scripteurs novice/experts, entre écrits et représentations orales des compétences scripturales. Dans leur article comparatif entre novice-étudiant et expert-chercheur, K. FlØtum et E. T. Vold montrent que les doctorants sont plus effacés énoncia-tivement que les chercheurs experts mais apparaissent plus appliqués dans la rédaction du processus de recherche respectant les normes du genre.

6 De même, les objets précis d'investigation sont nombreux: pronoms personnels marquant le positionnement de l'auteur, patrons phraséologiques dont la forme passive, les formes métonymiques ou des formes lexicales particulières. Ainsi les head nouns, «noms-tête » que I. Kanté présente ainsi : « The présence writers or speakers in their discourse through certain types of head nouns, such as fact, statement, assumption, etc. » et qui ont la particularité d'imposer l'emploi de " that ».

7 Nous nous arrêterons sur les formes marquant le positionnement de l'auteur scientifique. Sur cette question précise et souvent traitée dans le champ du discours scientifique, nous constatons que différentes formes de manifestation de l'auteur dans l'article de recherche sont envisagées à travers l'ensemble de ce volume. Trois types, selon Kjersti Fløtum et Eva Thue Vold (p. 44) qui distinguent les « rôles de scripteur, chercheur et argumentateur». Ces rôles permettent "d'affiner la notion de subjectivité en distinguant différents types de présence de l'auteur, certains plus «subjectifs » que d'autres. »

8 A. Tutin passe en revue les différentes formes linguistiques que peut prendre l'implication explicite de l'auteur (p. 18) :

- les pronoms personnels qui permettent de retrouver l'auteur-agent : je, nous et on. A ce sujet, U. Reutner contextualise, historiquement et culturellement le tabou initial du je-moi comme auteur scientifique et montre que son admission est aujourd'hui « perçue comme signe de sincérité ». De fait, elle y voit une "redéfinition de la notion de modestie: au lieu de faire étalage d'une modestie traditionnelle consistant dans la négation de soi-même au sens d'une captatio, l'auteur se nomme sciemment dans son texte et montre une nouvelle forme dépersonnalisante 厨...區 en étant prêt à en assumer 
la responsabilité » (p. 81). A. Tutin (p. 22) montre que le "nous de modestie, reste la norme dans les écrits scientifiques pour le français 監...血 contrairement à l'anglais où le I est prédominant. »

9 On y voit une ambigüité avec le on qui peut soit inclure le je, soit l'exclure, une ambigüité avec le nous exclusif $(=j e)$ et le nous hortatif, c'est à dire nous inclusif ayant effet d'impératif unissant le lecteur à l'auteur. (U Reutner, p. 93).

10 Les pronoms sujets renvoyant à l'auteur restent peu employés, «ce qui semblerait alimenter la thèse des écrits scientifiques comme un genre à fort effacement énonciatif, où la voix de l'auteur n'est pas mise en avant (au maximum, en linguistique, 13\% des occurrences lexicales)». Les auteurs constatent de grandes différences entre différentes disciplines des sciences humaines (A. Tutin, p. 25-26).

11 - Les verbes de positionnement se présentent selon A. Tutin (p. 24) sous trois catégories : verbes d'opinion ou de point de vue, verbes de choix et d'intention, verbes signifiant un apport spécifique. La conclusion (p. 31) est surprenante : « la majorité des verbes employés avec les pronoms personnels auteurs ne sont pas des verbes qui manifestent des prises de position marquées. L'auteur apparait essentiellement pour décrire les procédures scientifiques et accompagner le processus d'écriture ».

Par ailleurs certains verbes renvoient aux processus d'analyse accomplie par l'auteur, d'autres renvoient au processus même de l'écriture scientifique (ATutin, p. 29).

Ce numéro 41 de Lidil enrichira les données de tout chercheur s'intéressant à l'écriture scientifique, que ce soit du point de vue du processus d'écriture et du positionnement épistémique d'auteur ou des marques linguistiques spécifiques dont ils sont le champ d'expression.

\section{AUTEURS}

IRÈNE FENOGLIO

ITEM, CNRS 